\title{
Ghost lineages invalidate or reverse several results on gene flow
}

\author{
Théo Tricou' ${ }^{1}$ Eric Tannier ${ }^{1,2}$ and Damien M. de Vienne ${ }^{1, *}$ \\ ${ }^{1}$ Univ Lyon, Université Lyon 1, CNRS, Laboratoire de Biométrie et Biologie Évolutive UMR5558, \\ F-69622 Villeurbanne, France \\ ${ }^{2}$ INRIA Grenoble Rhône-Alpes, F-38334 Montbonnot, France
}

*Corresponding authors: E-mail: damien.de-vienne@univ-lyon1.fr

\begin{abstract}
Introgression, endosymbiosis and gene transfer, i.e. Horizontal Gene Flow (HGF), are primordial sources of innovation in all domains of life. Our knowledge on HGF relies on detection methods that exploit some of its signatures left on extant genomes. One of them is the effect of HGF on branch lengths of constructed phylogenies. This signature has been formalized in statistical tests for HGF detection, and used for example to detect massive adaptive gene flows in malaria vectors or to order evolutionary events involved in eukaryogenesis. However these studies rely on the assumption that ghost lineages (all unsampled extant and extinct taxa) have little influence. We demonstrate here with simulations and data re-analysis, that when considering the more realistic condition that unsampled taxa are legion compared to sampled ones, the conclusion of these studies become unfounded or even reversed. This illustrates the necessity to recognize the existence of ghosts in evolutionary studies.
\end{abstract}

\section{Introduction}

Gene flow between taxa affects all domains of Life, and occurs between taxa separated by different evolutionary time-scales: from introgression between populations to trans-phylum endosymbiosis. Introgression, where gene flow results from hybridization followed by repeated back-crossing with one of the parent species, is recognized as a major source of genetic variation in natural populations, that contributed to adaptation and adaptive radiation in most plant and animal groups, including humans (see Edelman and Mallet 2021 for a recent review). Horizontal Gene Transfer (HGT), where gene flow occurs between distinct taxon in a non-vertical manner and is mediated by 
mechanisms as diverse as natural transformation, transduction, conjugation, recombination or endosymbiosis (Thomas and Nielsen 2005; Keeling and Palmer 2008), appears now as a primary source of innovation in most taxonomic groups. Emblematic cases go from the acquisition of antibiotic resistance genes in bacteria (Davies and Davies 2010), to the emergence of mitochondria and chloroplasts in eukaryotic cells by endosymbiosis (Archibald 2015).

These events, hereafter referred to as horizontal gene flow (HGF) can be detected with phylogenetic approaches. Signature of HGF may be seen when phylogenies reconstructed from portions of the genome that were horizontally acquired (from single nucleotide to full chromosomes) contradict the evolutionary history of the taxa analyzed (represented by the species tree), and/or show differences in branch lengths. Indeed, the genetic (and thus phylogenetic) distance between the lineages involved in the flow (donor and recipient) for the horizontally acquired sequence reflect the time since the flow, not the time since their speciation.

These simple expectations form the basis of numerous studies of HGF, either to propose new methods for the detection of gene flow (Adato et al. 2015; Rosenzweig et al. 2016; Dalquen et al. 2017; Hahn and Hibbins 2019; Pfeifer et al. 2020; Forsythe et al. 2020b; Suvorov et al. 2021), or to resolve evolutionary puzzles in various taxonomic groups (Fontaine et al. 2015; Pittis and Gabaldón 2016; Forsythe et al. 2020a; Vosseberg et al. 2020; Suvorov et al. 2021).

Here, we focus specifically on the link between HGF and phylogenetic tree branch lengths. We show that the way branch lengths are interpreted in several important studies of gene flow overlooks the possible impact of ghost lineages on the expectations given above. By ghost lineages, we mean any taxon that is absent from the analysis, but is susceptible to interfere with the analyzed taxa via HGF. This means the extant taxa that are known but not sampled, extant taxa that are unknown, and all extinct taxa. More than $99.9 \%$ of all species that have ever lived are now extinct (Raup 1991), and the number of extant species that are still uncatalogued is almost an order of magnitude higher than those that have been reported (around 1.3 out of 8.7 Million eukaryotes estimated in 2011, Mora et al. 2011), or many orders of magnitudes higher according to some predictions (Locey and Lennon 2016). The amount of ghost taxa in any analysis of HGF is thus potentially huge.

We reanalyzed recent studies that used the expected link between HGF and branch lengths in different contexts: for the detection of introgressed loci (the $\mathrm{D}_{3}$ method, Hahn and Hibbins 2019), 
for resolving the correct branching order of Anopheles species (Fontaine et al. 2015) and for ordering the acquisitions of genes associated with the emergence of eukaryotic cells (Pittis and Gabaldón 2016; Vosseberg et al. 2020) and the elaboration of the chloroplast membrane (Sato 2020). Using simulations involving species trees with extinctions and gene trees prone to HGF, we show that under the realistic situation where most past and present taxa are ghosts, the branch length argument in relation to HGF is misleading, and can even lead to conclusions that are the opposite of the published ones. We emphasize the need to take into account ghost lineages in evolutionary studies and propose an alternative hypothesis when using branch lengths in phylogenies to investigate HGF: any signal of HGF should be interpreted in the first instance as an indication of the presence of ghost lineages that acted as donors, and not as an indication of a gene flow between lineages present in the analysis.

\section{Results}

\section{Preamble}

The three families of examples we explored in this study (the three next sections) illustrate the same principle regarding the impact of ghost lineages on phylogenetic tree branch lengths after HGF: the transfer of genetic material between lineages, that always decreases the genetic distance between the donor and the recipient when looking at the transferred region, may lead to an increase of the genetic distance between the lineages studied if the donor of the transfer (or its descendants) is absent from the analysis (i.e. it is a ghost lineage). This occurs for donor ghost lineages located outside the ingroup formed by the lineages studied. This is illustrated in Figure 1. While HGF between species present in the analysis produces phylogenetic gene trees with shorter branch lengths (scenarios A and B), HGF from a ghost lineage outside the ingroup (lineage "X") produces a gene tree with longer branches (scenario C). As a consequence, it might be wrong to consider that shorter branches are the result of gene flow, when in fact these shorter branches stem from the absence of introgression. We use simulations to assess the likelihood of these two opposite interpretations in different contexts. 

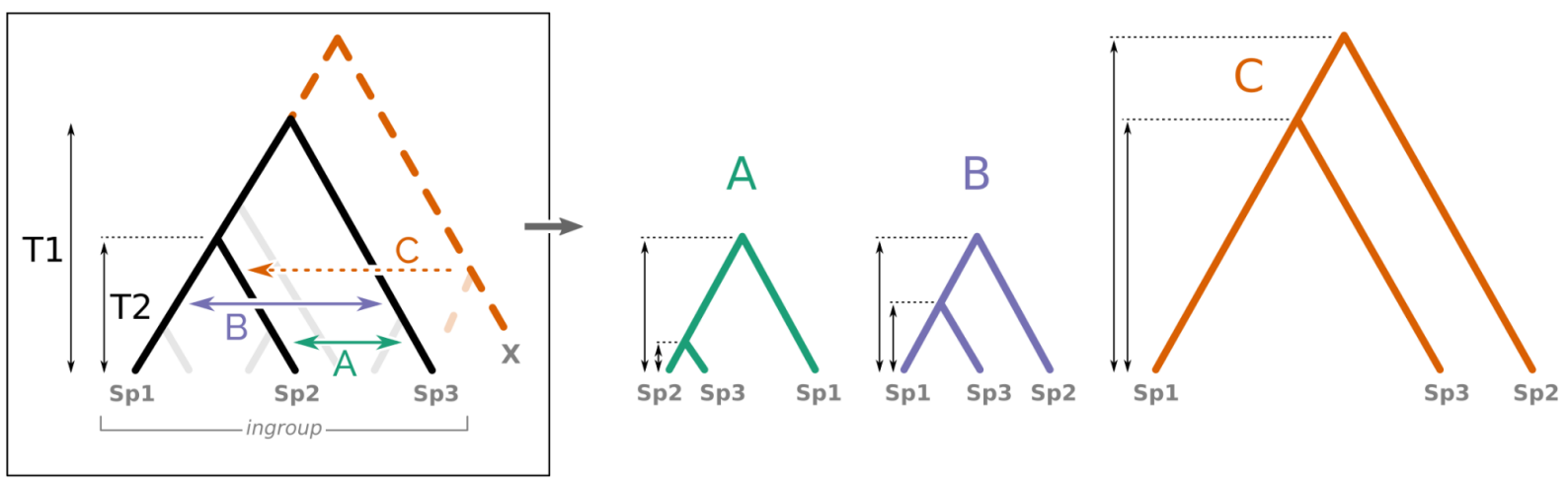

Figure 1. Effect of horizontal gene flow (HGF) on branch lengths in phylogenetic trees of the horizontally transferred genomic regions. HGF from a ghost lineage (X) from outside an ingroup comprising three lineages produces a phylogenetic tree with increased branch lengths compared to the species tree (scenario $\mathrm{C}$ ), while branch lengths are shorter when HGF occurs within the ingroup (scenarios A and B).

\section{Using branch lengths to detect introgression events (the $D_{3}$ method) is often misleading}

$\mathrm{D}_{3}$ (Hahn and Hibbins 2019) is a recent statistical method that uses branch lengths (i.e. pairwise distances between species) to detect introgression in a three-taxon tree. Referring to the notations in Figure 1, the test is aimed at detecting gene flow between sp2 and sp3 or sp1 and sp3 (cases A and $\mathrm{B}$, respectively), by computing the statistics:

$$
D_{3}=\frac{d_{s p 2-s p 3}-d_{s p 1-s p 3}}{d_{s p 2-s p 3}+d_{s p 1-s p 3}}
$$

Where $\boldsymbol{d}$ represents the pairwise distance between the species, which can be obtained by a measure of genetic distance between their genome sequences (see Hahn and Hibbins, 2019 for details). According to the original description of the test, if no introgression occured, $\mathrm{D}_{3}$ is close to 0 regardless of the presence of Incomplete Lineage Sorting (ILS, a process where the retention of ancestral polymorphism in the populations can lead to gene trees that differ from the species tree); but in the case of gene flow, $\mathrm{D}_{3}$ can be either positive, revealing introgression between $\mathrm{sp} 1$ and $\mathrm{sp} 3$, or negative, revealing introgression between $\mathrm{sp} 2$ and sp3. The significance of the test is determined by comparison of the computed $D_{3}$ value with a distribution of $D_{3}$ values obtained from block-bootstrap replicates of the sequences.

Therefore the $\mathrm{D}_{3}$ method (Hahn and Hibbins 2019), as well as other methods based on the same principle (Adato et al. 2015; Rosenzweig et al. 2016; Hahn and Hibbins 2019; Forsythe et al. 2020b; Pfeifer et al. 2020; Suvorov et al. 2021) relies on the assumption that short branch lengths will be observed following introgression events. But what if the donor has no sampled descendants, 
which is probably the most frequent situation? For instance, if an introgression occurs between a taxon from outside the tree (e.g. taxon $\mathrm{X}$ in Figure 1) and, for example, sp2 (scenario C in Figure 1)? This introgression event increases the distance between $\mathrm{sp} 2$ and $\mathrm{sp} 3$ without affecting the distance between sp1 and sp3 and results in a positive $\mathrm{D}_{3}$ that is interpreted as gene flow between sp1 and sp3 even though neither is involved in the introgression. While it has been shown several times that other tests of introgression can be deceived by ghost lineages (Durand et al. 2011; Hibbins and Hahn 2021; Tricou et al. 2021), the scale of this problem for the $\mathrm{D}_{3}$ test is still unknown.

In practice, it is possible to estimate the probability that ghost introgressions lead to erroneous interpretations of the $\mathrm{D}_{3}$ statistic. To this end, we simulated random species trees (40 extant species) and random introgression events with the $m s$ software (see Material and Methods), assuming that the age of the root of the trees was compatible with introgressions occuring uniformly between the species in the trees, but incompatible with introgressions with species outside. This can be seen as a strict discretization of the known correlation between the probability of hybridization, and consequently of introgression, and the genetic distance between species (Edmands 2002; Mallet 2005; Chapman and Burke 2007; Montanari et al. 2014). We will see later why this is justified.

For all possible three-taxa samples where $\mathrm{D}_{3}$ was significantly different from 0 , we evaluated whether this result was imputable to (i) introgression within the group containing the three taxa of interest (the ingroup), even when the donor taxon was not the one directly involved (a donor ghost taxon, sister to the one sampled, can give the same signal, see Figure 1), or (ii) a "ghost introgression" (from outside the ingroup), resulting in an erroneous interpretation of the test. Unsurprisingly, the probability of erroneously interpreting the $\mathrm{D}_{3}$ statistic was high when the size of the ingroup was small relative to the total size of the tree, almost reaching $100 \%$ when the ingroup comprised less than $20 \%$ of all taxa in the tree (Figure 2). Biologically speaking, it seems realistic to consider that there are more taxa with whom introgression can occur outside than inside a given three-taxa clade, especially when the three taxa chosen are "closely related species", which is a condition for the test to be used (Hahn and Hibbins 2019).

Therefore we propose that any $\mathrm{D}_{3}$ statistic that is significantly different from 0 should be interpreted in the first instance as the result of an introgression event originating from outside the tree formed by the three taxa considered. 


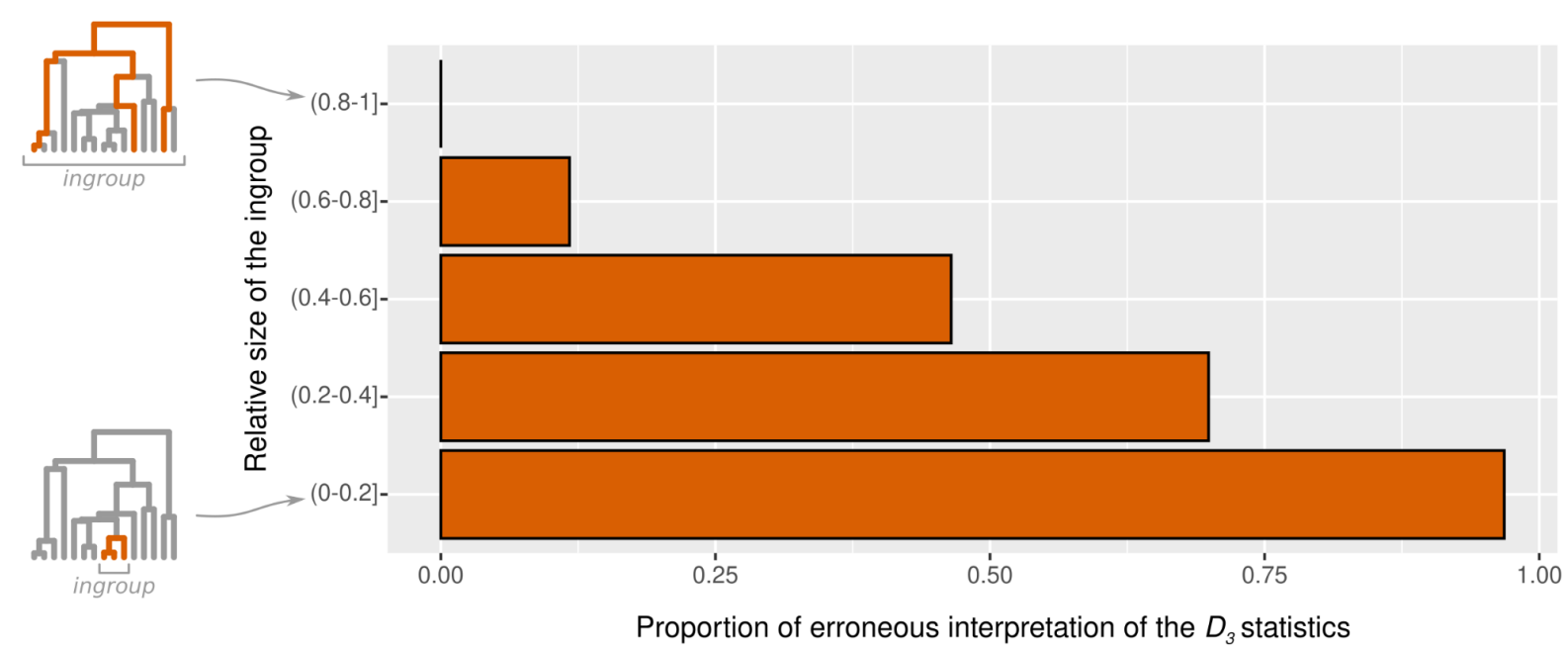

Figure 2. Proportion of cases (from simulations) where a $D_{3}$ test is significantly different from 0 because of an introgression from a ghost lineage from outside the ingroup. These cases may be incorrectly interpreted (see text) and lead to the misidentification of both taxa involved in the introgression. The proportion of erroneous interpretations of the test is computed for different relative sizes of the ingroup, i.e. the number of taxa of the smallest subtree containing the three taxa studied divided by the total number of taxa in the tree (see trees on the left).

\section{Genes with long branch lengths are not good markers of the mosquito species phylogeny}

It is common practice in phylogenetics to use gene markers that are supposed to not be horizontally transferred to construct the history of species diversification. This is important because HGF can change the branching structure (i.e. the topology) and the branch lengths of gene phylogenies (Figure 1). The assumption that HGF decreases branch lengths in gene phylogenies would suggest that among multiple possibilities of species tree topologies, the one similar to the gene trees with the longest branch lengths would represent the "true" species phylogeny because it agrees with genes that are expected to not have experienced gene flow.

This approach, i.e. finding the species tree supported by the genes with the longest branches, was proposed and used to recover the branching order of three Anopheles species, An. arabiensis, An. gambiae and An. coluzzii (Fontaine et al. 2015). However, as explained in the preamble, this hypothesis may be incorrect because, in the presence of ghost lineages, long branches may result from introgression and are not an indication of its absence.

To test the effect of ghost lineages on the strategy proposed by Fontaine et al. (2015) to find the correct branching pattern of three closely related species, we simulated a species tree from which three species were chosen (Figure 3, top). We chose three closely related species to mimic the three species of the original study, that belong to the Anopheles gambiae species complex, a group that 
diverged only 1.8 My ago (Fontaine et al. 2015) when the age of the Anopheles genera is dated between 25 and 93 My (Moreno et al. 2010; Logue et al. 2013; Zhao et al. 2013). Using ms, we simulated gene trees under two simple evolutionary scenarios (see Material and Methods): one involving introgression between ingroup species $\mathrm{B}$ and $\mathrm{C}$ (scenario 1), and one involving introgression from a ghost lineage from outside the ingroup to species B (scenario 2, Figure 3). We separated the simulated gene trees into two categories: those with a similar topology to the species tree and those with a discordant topology. We then computed for each category the mean divergence times (T1 and T2) for the nodes separating the three species (Figure 3).

We observed that under scenario 1, divergence times in gene trees with discordant topologies were on average shorter than in gene trees with the same topology as the species tree (Figure 3). This is in agreement with the expectations of the Anopheles study: "Because introgression will reduce sequence divergence between the species exchanging genes, we expect that the correct species branching order revealed by gene trees constructed from non introgressed sequences will show deeper divergences than those constructed from introgressed sequence" (Fontaine et al. 2015).

However, when introgression came from a ghost lineage from outside the ingroup (such as in scenario 2), we observed the opposite result, with gene trees with discordant topologies exhibiting longer divergence times than gene trees with the same topology as the species tree. In this case, it is incorrect to consider that the true species tree topology is the one supported by the genes with longest divergence times. 

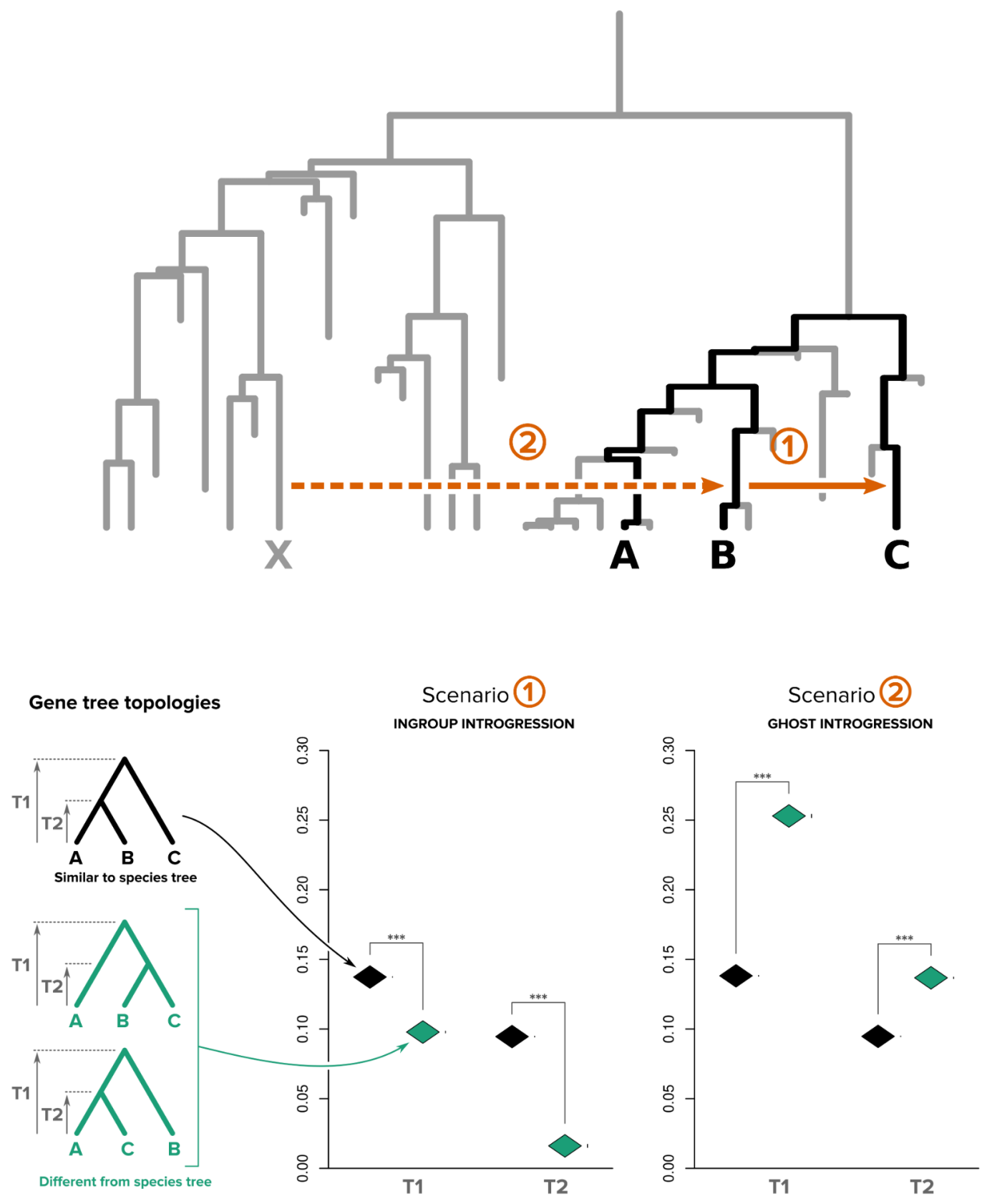

Figure 3. Impact of ghost lineages on the use of branch lengths to choose between alternative species trees. Top panel: the complete species tree, black branches represent the $\mathrm{ABC}$ tree while grey branches represent ghost lineages. Genome evolution (1,000 genes) was simulated under two different introgressions scenarios: scenario 1, ingroup introgression (solid orange arrow) between species $\mathrm{B}$ and $\mathrm{C}$, and scenario 2, ghost introgression (dotted orange arrow) between ghost lineage $\mathrm{X}$ and species B. Bottom panel. Left: the three possible topologies representing the relationship between species A,B and C. Right: The mean divergence times $\mathrm{T} 1$ and $\mathrm{T} 2$ were computed for both scenarios and for all genes supporting either the species tree topology $((\mathrm{A}, \mathrm{B}), \mathrm{C})$ (black rhombus) or the two discordant topologies $((\mathrm{B}, \mathrm{C}), \mathrm{A})$ and $((\mathrm{A}, \mathrm{C}), \mathrm{B})$ (green rhombus; whiskers standard error of the mean, *** for t-test with P-value $<2.2 \mathrm{e}-16$ ). 


\section{Using branch lengths to order acquisition events (the stem-length method) is misleading in the presence of ghost lineages}

Recent studies have used the so-called "stem-length method" to reconstruct the relative timing of acquisition of different genes by a proto-eukaryotic cell during eukaryogenesis (Pittis and Gabaldón 2016; Vosseberg et al. 2020). This method was also used to characterize the relative timing of acquisition of genes involved in the elaboration of the chloroplast membrane (Sato 2020). The stem-length method, shown in Figure 4, relies on the expectation that early acquisitions of genes by a given recipient lineage should result in longer branches (or stems) at the base of this lineage in the corresponding gene trees than late acquisitions (Figure 4, top). This approach was used to address the long-standing question of the early or late acquisition of mitochondria during eukaryogenesis (Pittis and Gabaldón 2016). This study concluded that the shorter stems in the trees of eukaryotic genes of alphaproteobacterial origin supported the latter.

However, if ghost lineages are taken into account, the expectations of the stem-length method can be totally reversed. If the donor lineage has no descendants, either because they all went extinct, because they have not been discovered, or because they were not considered in the study, then stem lengths will be determined not by the time when the transfer occurred but by the divergence time between the missing (ghost) clade from which the transfer originated and its closest sampled relative (Figure 4, bottom). Under these circumstances, the correlation between order of acquisition and stem-length can be reversed.

To quantify this effect and have an insight into the validity of the results using the stem length method, we performed a simulation where species trees with 1,000 leaves were generated following a birth-death process and pairs of acquisition events were repeatedly sampled in the tree to mimic early and late acquisitions. We then sampled a proportion of the extant species (between $1 \%$ and $10 \%$ of the total) and looked at the effect of sampling on the predictions of the stem-length method regarding the order of events (Figure 5). We observed that when 10\% of species were sampled, $\sim 33.5 \%$ of the predictions were wrong (predicting that event A occurred before event B when the opposite actually happened). This proportion reached almost 50\% (the maximum possible, equivalent to a random prediction) when $1 \%$ of the species were sampled (Figure 5A). 


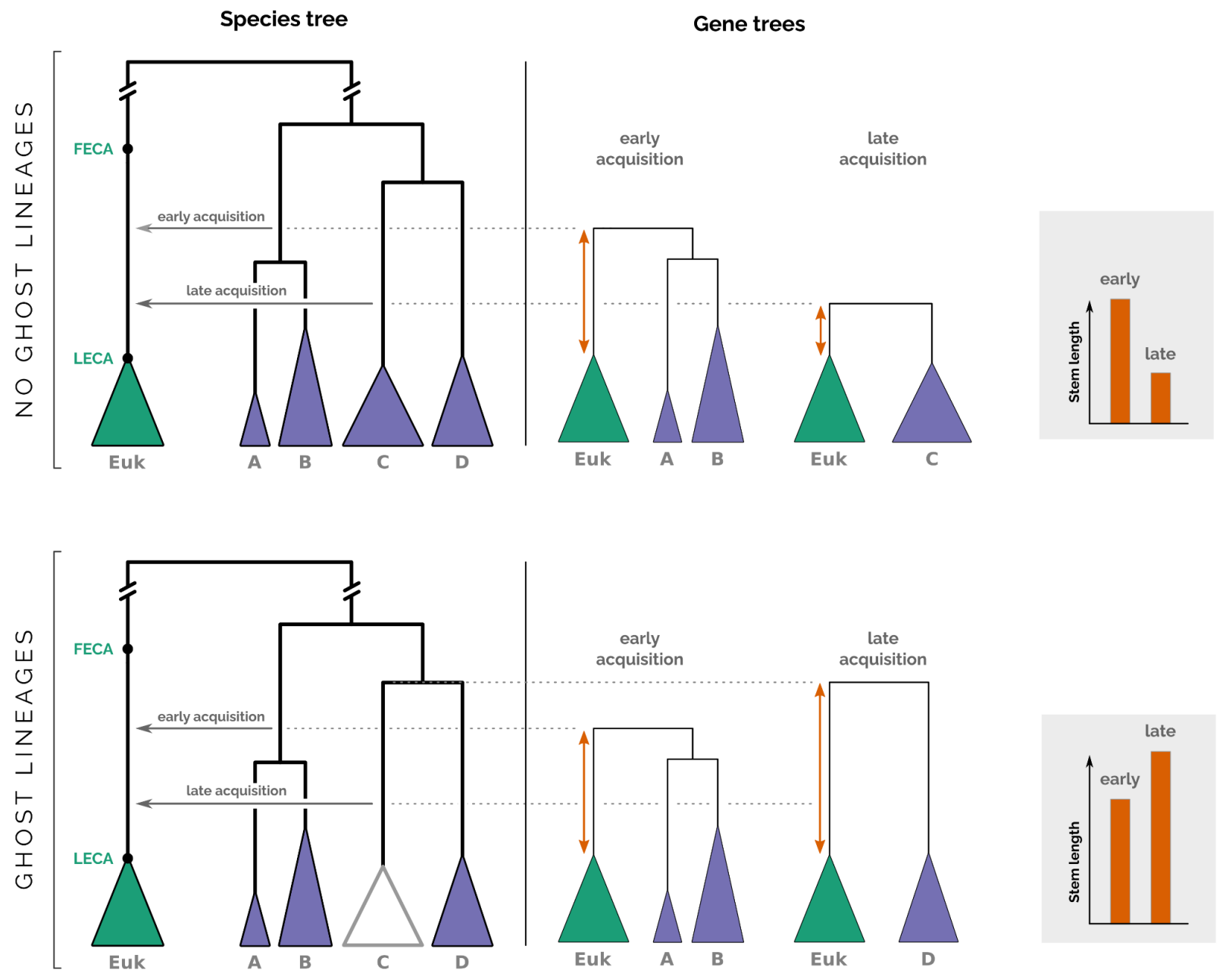

Figure 4. Illustration of the impact of ghost lineages on the relative timing of genomic acquisitions with the stem-length method. Under the hypothesis of all clades being sampled, i.e. there are no ghost lineages (top), an early acquisition produces a gene tree with a long stem, while a late acquisition produces a gene tree with a short stem (orange arrows show stem lengths, shown in the grey box on the right). If a clade is missing, i.e. there are ghost lineages (bottom), the opposite observation can be made. Because the donor lineage (here the ancestor of C) left no descendants, and because it split from the rest of the clade (D) before the time of the early acquisition event, the correlation between stem lengths and acquisition time is reversed: early acquisition leads to a shorter stem length than late acquisition (grey box).

We also observed that the error increased when the time interval between the two events was shorter. Indeed, nearly $41 \%$ of predictions were wrong when the time interval between events was less than $10 \%$ of the tree height with $10 \%$ of species sampled (Figure $5 \mathrm{~B}$ ). 

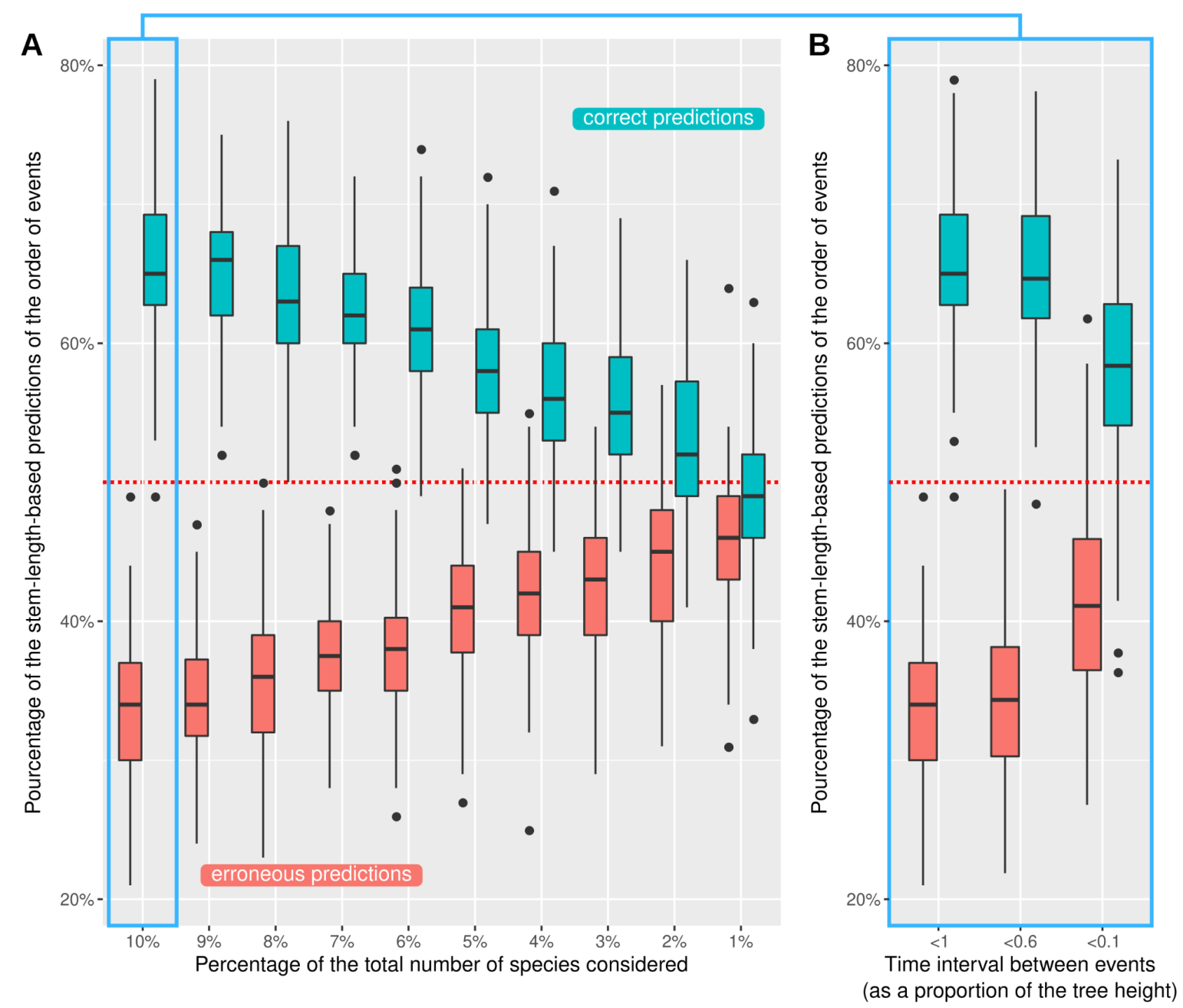

Figure 5. Effect of the proportion of species sampled (A) and the length of the time interval between acquisition events (B) on the percentage of right (blue bars) and wrong (red bars) predictions of the order of events using the stem-length method. The dashed red line represents what would be observed if predictions were random. Cases where the stem-length method could not order events (because stems had the same length) were removed, which explains why the blue and red bars do not add up to $100 \%$.

\section{Discussion}

Branch-length-based approaches are versatile methods for studying different aspects of horizontal gene flow (HGF) at different scales, from intraspecific introgression (between populations) to transphylum gene transfers. These approaches rely on the expectation that any HGF should result in shorter (phylo)genetic distances between donor and recipient in trees of the transferred genomic sequence(s) compared to trees of other (presumably vertically transferred) sequences 
from the same taxa. However, this only holds if all lineages, or at least the sister lineages of all lineages under consideration, are present in the study. Indeed, as demonstrated here, an apparent decrease in (phylo)genetic distance between two taxa can in actual fact result from an increase in (phylo)genetic distance between other (unsampled) taxa. Therefore, it appears crucial to take ghost lineages into account when interpreting the result of branch-length-based HGF inference methods.

The simulations performed here are simple, but they illustrate the impact that ghost lineages can have on approaches as diverse as the $\mathrm{D}_{3}$ test (Hahn and Hibbins 2019), the test developed for finding the correct branching order in species groups (Fontaine et al. 2015) and the stem-length method for ordering events (Pittis and Gabaldón 2016).

First, our simulations demonstrate that under the realistic assumption that there are many ghost lineages, not only does the likelihood that HGF comes from unknown species increase, but also the possibility that the identification of both the donor and the recipient of the transfer is erroneous, leading to the identification of two lineages that have nothing to do with this process. Thus, when interpreting the results of introgression tests like $\mathrm{D}_{3}$ (Hahn and Hibbins 2019), the possibility of introgression from ghost lineages from outside the three taxa considered should be systematically taken into consideration as a possible alternative scenario and should be considered to be (at least) as probable as the usual interpretation.

Second, we show that HGF from a ghost lineage could in some cases increase branch lengths in a gene tree, instead of decreasing them as is commonly expected. This absence of an unambiguous pattern suggests that branch lengths may not be suitable for identifying appropriate markers for phylogenetic reconstruction as was done in Fontaine et al. (2015). Introgressions are frequent in the Anopheles genera, so that the possibility of an introgression from an unsampled (known, unknown or extinct) species does not seem unlikely. If this is the case, the claim that the branching order of the species in Anopheles gambiae species complex is supported by a minority portion of the genome (the $\mathrm{X}$ chromosome) may well be wrong. The apparent "massive introgression" in autosomal regions could be due in fact to less frequent and massive introgression events in the $\mathrm{X}$ chromosomal region involving (as donor) a species that branches outside the ones considered in the study. 
Third, because HGF can increase or decrease branch lengths depending on the sampling effort and the proportion of ghost lineages, we show that the stem-length method that infers the relative timing of transfer events is prone to errors. To quantify this effect on biological data, it is necessary to answer the following questions regarding any clade where the stem-length method is to be applied: 1) How likely is it that the lineages from which the transfers originated have no descendants?, and 2) How far apart are the events under investigation, relative to the total timespan considered?

We can provide the following elements concerning the biological data for which the stem-length method was devised and on which it was applied originally (Pittis and Gabaldón 2016; Vosseberg et al. 2020). The acquisitions in question are genes of bacterial origin transferred to protoeukaryotes after the appearance of the First Eukaryotic Common Ancestor (FECA) and before that of the Last Eukaryotic Common Ancestor (LECA), i.e. between ca. 2.3-2.7 and ca. 1.1-1.2 BYA (reviewed in Chernikova et al. 2011). It is difficult to infer the macroevolutionary history of bacteria, especially at such deep evolutionary timescales, but it is clear that most lineages living during that period went extinct (Louca et al. 2018) and some went extinct during mass extinctions (Weinbauer and Rassoulzadegan 2007). In addition, it is clear that most extant bacterial lineages are still unknown (Locey and Lennon 2016; Louca et al. 2018) and may not be scattered uniformly across the known diversity. Indeed, unsampled lineages are likely to form major clades, as illustrated by the discovery of a complete new phylum (CPR) in 2016 (Hug et al. 2016).

Given that only a small fraction of the diversity (extinct and extant) is known, we may consider the stem-length method as equivalent to a random or arbitrary choice of the relative timing of acquisition events.

In conclusion, we highlight here the fact that most HGF-detection results are interpreted without considering ghost lineages. Failure to consider the impact of ghost lineages on the interpretation of such tests impair their usefulness and lead to erroneous conclusions. As such, and considering the huge number of invisible lineages in real life, we suggest that the results of gene flow-related methods should be interpreted with the signature of ghost lineages as the foremost hypothesis. 


\section{Material and Methods}

\section{The effect of ghost lineages on the $D_{3}$ test for introgression}

\section{Simulating species trees and introgressions}

200 random species trees with a birth-death model were simulated using Zombi (Davín et al. 2020). Speciation and extinction rates were fixed at 1 and 0.9 , respectively. Simulations were run until 40 extant species were reached then 20 species were sampled. We converted the topology of the species tree into a suitable format for the coalescent simulator ms (Hudson 2002) with a custom python script: branch lengths were converted into units of generation and the age of the root of the trees was fixed at $10^{6}$ generations. To simulate introgression, a single migration event was imposed over one generation for a fraction $\mathbf{f}=50 \%$ of the donor population targeting the recipient population. This migration rate was used to ensure that introgression detection using $\mathrm{D}_{3}$ would not be biased by false positives. Then, for each species tree, we used $m s$ implemented in the R package Coala (Staab and Metzler 2016) to simulate 1,000 gene trees evolving in populations of fixed size (Ne) of 100,000 individuals.

\section{Computing the $D_{3}$ statistic}

To compute the $\mathrm{D}_{3}$ statistic for three lineages with the species tree topology ((P1,P2),P3), we calculated $\mathrm{D}_{13}$ and $\mathrm{D}_{23}$, the sum of the distance (or branch length) separating P1 and P3 and P2 and $\mathrm{P} 3$, respectively, across all gene trees $\left(1,000\right.$ in total). We then computed the $\mathrm{D}_{3}$ statistic using equation (1). This was done for each trio of lineages $((\mathrm{P} 1, \mathrm{P} 2), \mathrm{P} 3)$ in each species tree simulated. $\mathrm{D}_{3}$ significance was tested by bootstrap resampling of 1,000 gene trees with 1,000 replicates. We then calculated the $Z$-score and considered that $\mathrm{D}_{3}$ was significant if $\mathrm{Z}>3$ or $\mathrm{Z}<$ 3, following Green et al. (2010). Finally, as we tracked the true donor and recipient lineages in each simulation, we assessed for each $\mathrm{D}_{3}$ whether the test was significant due to an ingroup introgression or a ghost introgression event from outside the three species considered.

\section{Using branch lengths to determine the correct species tree topology Species tree simulation with ingroup or ghost introgression}

A species tree was simulated using Zombi (Davín et al. 2020). Unless otherwise stated, parameters were the same as the one used in the previous section. The simulation was run until 
16 extant species were reached. On this tree, three species with the topology $((\mathrm{A}, \mathrm{B}), \mathrm{C})$ were arbitrarily chosen. All others were considered to be ghost lineages. We then converted the topology of the tree into an $m s$ readable tree for coalescent simulation. Two datasets were generated with $m s$; in both datasets the number of generations separating the tip from the root of the tree was fixed to $5 \times 10^{6}$ and a migration event was imposed for a fraction $f=20 \%$. For the first dataset, migration took place from $\mathrm{B}$ to $\mathrm{C}$, i.e. between two extant species (ingroup introgression). For the second dataset, migration took place between a randomly sampled ghost lineage outside of the triplet phylogeny and B (ghost introgression). For both models, we simulated 1,000 gene trees evolving in populations of fixed size $(\mathrm{Ne})$ of 100,000 individuals.

\section{Branch length in gene trees.}

We identified the gene trees that were congruent with the $((A, B), C)$ topology (corresponding to the branching order of the species tree) and those that corresponded to one of the two discordant topologies $((\mathrm{B}, \mathrm{C}), \mathrm{A})$ or $((\mathrm{A}, \mathrm{C}), \mathrm{B})$ that can arise from ILS or introgression. Subsequently, for both introgression models described above and for each gene tree, we computed the value of species divergence times T1 and T2 (see Figure 3) following the equation from Fontaine et al. (2015) (see supplementary material S3.2 in Fontaine et al. (2015)).

\section{The effect of ghost lineages on the inference of the relative timing of gene acquisitions using the stem-length method}

The order of acquisition events from simulated trees with and without ghost lineages was compared to the predicted order of events using the stem-length method (Pittis and Gabaldón 2016; Vosseberg et al. 2020). Simulations were carried out as follows:

- $\quad 100$ trees under a birth-death process (speciation rate $=1$, extinction rate $=0.5$ ) were generated using the rphylo function in the R package ape (Paradis and Schliep 2019); simulations were run until trees reached 1,000 leaves.

- $\quad$ Two points were randomly sampled from each tree representing two origins of transfer (or acquisitions) towards the same recipient lineage; we recorded their timing and the time interval $(d t)$ between them (as a fraction of the total tree height).

- $\quad$ Trees were pruned by sampling a proportion $p$ of the leaves from each tree.

- We evaluated the new order of events on the pruned tree using the stem-length method. 
- We recorded whether the order of events before and after pruning was the same (1), was different $(0)$ or could not be determined (i.e. events occurred at the same time) (NA). The latter occurs if the two events lead to equal stem lengths after sampling.

The proportion of each $(1,0, \mathrm{NA})$ out of the 100 replicates was calculated.

The proportion of sampled leaves $p$ ranged between 1\% (10 leaves) and 10\% (100 leaves), in 1\% increments. We performed 100 replicates to obtain a variance for the observed proportions of correct and erroneous predictions of the order of events.

To explore the effect of the time interval $(d t)$ between events on the proportion of erroneous predictions, we subsampled the pairs of events with $d t<1, d t<0.6$ and $d t<0.1$ and recomputed the proportion of correct and erroneous predictions each time. This analysis was carried out with $p=10 \%$.

\section{Acknowledgments}

We thank Adrián A. Davín for sharing his insight on performing simulations and Gergely J. Szöllősi for useful discussions. This work was supported by the French National Research Agency (Grants ANR-18-CE02-0007-01 and ANR-19-CE45-0010). Simulations were performed using the computing facilities of the CC LBBE/PRABI.

\section{Software Availability}

All codes used to generate and analyze the simulations performed in this study are available at: https://github.com/theotricou/Ghost_branch_length.git.

\section{References}

Adato O., Ninyo N., Gophna U., Snir S. 2015. Detecting Horizontal Gene Transfer between Closely Related Taxa. PLOS Comput. Biol. 11:e1004408.

Archibald J.M. 2015. Endosymbiosis and Eukaryotic Cell Evolution. Curr. Biol. CB. 25:R911-921.

Chapman M.A., Burke J.M. 2007. Genetic divergence and hybrid speciation. Evol. Int. J. Org. Evol. 61:1773-1780.

Chernikova D., Motamedi S., Csürös M., Koonin E.V., Rogozin I.B. 2011. A late origin of the extant eukaryotic diversity: divergence time estimates using rare genomic changes. Biol. Direct. 6:26.

Dalquen D.A., Zhu T., Yang Z. 2017. Maximum Likelihood Implementation of an Isolation-with-Migration Model for Three Species. Syst. Biol. 66:379-398. 
Davies J., Davies D. 2010. Origins and Evolution of Antibiotic Resistance. Microbiol. Mol. Biol. Rev. MMBR. 74:417-433.

Davín A.A., Tricou T., Tannier E., de Vienne D.M., Szöllősi G.J. 2020. Zombi: a phylogenetic simulator of trees, genomes and sequences that accounts for dead linages. Bioinformatics. 36:1286-1288.

Durand E.Y., Patterson N., Reich D., Slatkin M. 2011. Testing for Ancient Admixture between Closely Related Populations. Mol. Biol. Evol. 28:2239-2252.

Edelman N.B., Mallet J. 2021. Prevalence and Adaptive Impact of Introgression. Annu. Rev. Genet. 55.

Edmands S. 2002. Does parental divergence predict reproductive compatibility? Trends Ecol. Evol. 17:520-527.

Fontaine M.C., Pease J.B., Steele A., Waterhouse R.M., Neafsey D.E., Sharakhov I.V., Jiang X., Hall A.B., Catteruccia F., Kakani E., Mitchell S.N., Wu Y.-C., Smith H.A., Love R.R., Lawniczak M.K., Slotman M.A., Emrich S.J., Hahn M.W., Besansky N.J. 2015. Extensive introgression in a malaria vector species complex revealed by phylogenomics. Science. 347.

Forsythe E.S., Nelson A.D.L., Beilstein M.A. 2020a. Biased gene retention in the face of introgression obscures species relationships. Genome Biol. Evol.

Forsythe E.S., Sloan D.B., Beilstein M.A. 2020b. Divergence-Based Introgression Polarization. Genome Biol. Evol. 12:463-478.

Green R.E., Krause J., Briggs A.W., Maricic T., Stenzel U., Kircher M., Patterson N., Li H., Zhai W., Fritz M.H.-Y., Hansen N.F., Durand E.Y., Malaspinas A.-S., Jensen J.D., Marques-Bonet T., Alkan C., Prüfer K., Meyer M., Burbano H.A., Good J.M., Schultz R., Aximu-Petri A., Butthof A., Höber B., Höffner B., Siegemund M., Weihmann A., Nusbaum C., Lander E.S., Russ C., Novod N., Affourtit J., Egholm M., Verna C., Rudan P., Brajkovic D., Kucan Ž., Gušic I., Doronichev V.B., Golovanova L.V., Lalueza-Fox C., Rasilla M. de la, Fortea J., Rosas A., Schmitz R.W., Johnson P.L.F., Eichler E.E., Falush D., Birney E., Mullikin J.C., Slatkin M., Nielsen R., Kelso J., Lachmann M., Reich D., Pääbo S. 2010. A Draft Sequence of the Neandertal Genome. Science. 328:710-722.

Hahn M.W., Hibbins M.S. 2019. A Three-Sample Test for Introgression. Mol. Biol. Evol. 36:2878-2882.

Hibbins M., Hahn M. 2021. Phylogenomic approaches to detecting and characterizing introgression. .

Hudson R.R. 2002. Generating samples under a Wright-Fisher neutral model of genetic variation. Bioinformatics. 18:337-338.

Hug L.A., Baker B.J., Anantharaman K., Brown C.T., Probst A.J., Castelle C.J., Butterfield C.N., Hernsdorf A.W., Amano Y., Ise K., Suzuki Y., Dudek N., Relman D.A., Finstad K.M., Amundson R., Thomas B.C., Banfield J.F. 2016. A new view of the tree of life. Nat. Microbiol. 1:1-6.

Keeling P.J., Palmer J.D. 2008. Horizontal gene transfer in eukaryotic evolution. Nat. Rev. Genet. 9:605-618. 
Locey K.J., Lennon J.T. 2016. Scaling laws predict global microbial diversity. Proc. Natl. Acad. Sci. 113:5970-5975.

Logue K., Chan E.R., Phipps T., Small S.T., Reimer L., Henry-Halldin C., Sattabongkot J., Siba P.M., Zimmerman P.A., Serre D. 2013. Mitochondrial genome sequences reveal deep divergences among Anopheles punctulatus sibling species in Papua New Guinea. Malar. J. 12:64.

Louca S., Shih P.M., Pennell M.W., Fischer W.W., Parfrey L.W., Doebeli M. 2018. Bacterial diversification through geological time. Nat. Ecol. Evol. 2:1458-1467.

Mallet J. 2005. Hybridization as an invasion of the genome. Trends Ecol. Evol. 20:229-237.

Montanari S.R., Hobbs J.-P.A., Pratchett M.S., Bay L.K., Van Herwerden L. 2014. Does genetic distance between parental species influence outcomes of hybridization among coral reef butterflyfishes? Mol. Ecol. 23:2757-2770.

Mora C., Tittensor D.P., Adl S., Simpson A.G.B., Worm B. 2011. How Many Species Are There on Earth and in the Ocean? PLoS Biol. 9:e1001127.

Moreno M., Marinotti O., Krzywinski J., Tadei W.P., James A.A., Achee N.L., Conn J.E. 2010. Complete mtDNA genomes of Anopheles darlingi and an approach to anopheline divergence time. Malar. J. 9:127.

Paradis E., Schliep K. 2019. ape 5.0: an environment for modern phylogenetics and evolutionary analyses in R. Bioinformatics. 35:526-528.

Pfeifer B., Alachiotis N., Pavlidis P., Schimek M.G. 2020. Genome scans for selection and introgression based on k-nearest neighbour techniques. Mol. Ecol. Resour. 20:1597-1609.

Pittis A.A., Gabaldón T. 2016. Late acquisition of mitochondria by a host with chimaeric prokaryotic ancestry. Nature. 531:101-104.

Raup D.M. 1991. Extinction: bad genes or bad luck? New York: W.W. Norton.

Rosenzweig B.K., Pease J.B., Besansky N.J., Hahn M.W. 2016. Powerful methods for detecting introgressed regions from population genomic data. Mol. Ecol. 25:2387-2397.

Sato N. 2020. Complex origins of chloroplast membranes with photosynthetic machineries: multiple transfers of genes from divergent organisms at different times or a single endosymbiotic event? J. Plant Res. 133:15-33.

Staab P.R., Metzler D. 2016. Coala: an R framework for coalescent simulation. Bioinformatics. 32:1903-1904.

Suvorov A., Kim B.Y., Wang J., Armstrong E.E., Peede D., D’Agostino E.R.R., Price D.K., Wadell P., Lang M., Courtier-Orgogozo V., David J.R., Petrov D., Matute D.R., Schrider D.R., Comeault A.A. 2021. Widespread introgression across a phylogeny of 155 Drosophila genomes. bioRxiv.:2020.12.14.422758.

Thomas C.M., Nielsen K.M. 2005. Mechanisms of, and Barriers to, Horizontal Gene Transfer between Bacteria. Nat. Rev. Microbiol. 3:711-721.

Tricou T., Tannier E., Vienne D.M. de. 2021. Ghost lineages highly influence the interpretation of introgression tests. :2021.03.30.437672.

Vosseberg J., van Hooff J.J.E., Marcet-Houben M., van Vlimmeren A., van Wijk L.M., Gabaldón T., Snel B. 2020. Timing the origin of eukaryotic cellular complexity with ancient 
duplications. Nat. Ecol. Evol.:1-9.

Weinbauer M., Rassoulzadegan F. 2007. REVIEW: Extinction of microbes: evidence and potential consequences. Endanger. Species Res. 3:205-215.

Zhao Z., Su T.-J., Chesters D., Wang S., Ho S.Y.W., Zhu C.-D., Chen X.-L., Zhang C.-T. 2013. The mitochondrial genome of Elodia flavipalpis Aldrich (Diptera: Tachinidae) and the evolutionary timescale of Tachinid flies. PloS One. 8:e61814. 